\title{
AN OPEN MAPPING THEOREM ON HOMOGENEOUS SPACES
}

\section{SHOZO KOSHI and MASAMICHI TAKESAKI}

(Received 22 June 1990)

Communicated by S. Yamamuro

\begin{abstract}
We shall prove an open mapping theorem concerning a Polish group acting transitively on a complete metric space.
\end{abstract}

1991 Mathematics subject classification (Amer. Math. Soc.): 46 A 30, 54 C 10.

Let $X$ be a topological space and $G$ be a topological transformation group on $X$ which is a Polish (separable, complete metric) group acting transitively on $X$. This action is denoted by the map $\psi$ from $G \times X$ to $X$ with $\psi(g, x)=g \cdot x$ for $g \in G$ and $x \in X$.

We shall consider an open mapping theorem on the map $G \ni g \rightarrow$ $\psi(g, x)=g \cdot x \in X$ from $G$ to $X$ for each fixed $x \in X$.

Even if there is a famous open mapping theorem on Banach spaces or topological linear spaces, the proof of this case is different from that of Banach space cases.

We shall call $\psi$ continuous if $\psi$ is continuous as the map from $G \times X$ to $X$. We shall call $\psi$ separately continuous if

(1) for each fixed $g \in G$, the map $x \rightarrow g \cdot x$ from $X$ to $X$ is continuous,

(2) for each fixed $x \in X$, the map $g \rightarrow g \cdot x$ from $G$ to $X$ is continuous. Now, we have the following theorem.

This work was done during a stay of the second author at Hokkaido University, by support of JSPS.

(C) 1992 Australian Mathematical Society 0263-6115/92 \$A2.00+0.00 
THEOREM A. Let $G$ be a complete metric group acting on a metric space $X$. If the map $\Psi: G \times X \rightarrow X$ defined by $\Psi(g, x)=g \cdot x$ is separately continuous, then $\Psi$ is continuous.

Proof. Due to the inequality:

$$
d\left(g x, g_{0} x_{0}\right) \leq d\left(g x, g x_{0}\right)+d\left(g x_{0}, g_{0} x_{0}\right),
$$

the continuity of $\psi$ at $\left(g_{0}, x_{0}\right) \in G \times X$ follows from the claim that for any $\varepsilon>0$ there exist $\delta>0$ and a neighborhood $U$ of $g_{0}$ such that $d\left(g x, g x_{0}\right)<\varepsilon$ whenever $d\left(x, x_{0}\right)<\delta$ and $g \in U$. Fix $x_{0} \in X$, and set

$$
A_{m, n}=\left\{g \in G: d\left(g x, g x_{0}\right) \leq \frac{1}{m} \text { if } d\left(x, x_{0}\right)<\frac{1}{n}\right\} .
$$

The separate continuity of $\psi$ yields the closedness of each $A_{m, n}$ and $G=$ $\bigcup_{n=1}^{\infty} A_{m, n}$. Furthermore, $A_{m, n} \subset A_{m, n+1}$ by construction. Baire's category property of $G$ implies that every non-empty open subset $U$ of $G$ contains a non-empty open subset $V \subset U$ such that $V \subset A_{m, n(m)}$ for some $n(m) \in \mathbb{N}$. Thus, we can find a sequence of open subsets $O_{m}$ of $G$ such that

$$
\begin{gathered}
O_{m} \subset A_{m, n(m)}, \text { diameter }\left(O_{m}\right)<1 / m, \\
O_{m} \supset \bar{O}_{m+1} .
\end{gathered}
$$

By the completeness of $G$, there exists a point $g_{0} \in \bigcap_{m=1}^{\infty} O_{m}$. It then follows that $d\left(g x, g x_{0}\right) \leq 1 / m$ if $d\left(x, x_{0}\right)<1 / n(m)$ and $g \in O_{m}$. Hence $\psi$ is continuous at $\left(g_{0}, x_{0}\right)$ by the first observation. Therefore, for any $\varepsilon_{1}>0$ there exists $\delta>0$ and a neighborhood $U$ of $g_{0}$ such that $d\left(g x, g_{0} x_{0}\right)<\varepsilon_{1}$ whenever $g \in U$ and $d\left(x, x_{0}\right)<\delta$. for an arbitrary $g_{1} \in G$ and $\varepsilon>0$, choose $\varepsilon_{1}>0$ so that $d\left(g_{1} g_{0}^{-1} y, g_{1} x_{0}\right)<\varepsilon$ whenever $d\left(y, g_{0} x_{0}\right)<\varepsilon_{1}$. We then set $V=g_{1} g_{0}^{-1} U$ as a neighborhood of $g_{1}$ and conclude that if $g \in V$ and $d\left(x, x_{0}\right)<\delta$ then $g_{0} g_{1}^{-1} g \in U$ so that $d\left(g_{0} g_{1}^{-1} g x, g_{0} x_{0}\right)<\varepsilon_{1}$ and therefore

$$
d\left(g x, g_{1} x_{0}\right)<\varepsilon \text { if } g \in V \text { and } d\left(x, x_{0}\right)<\delta .
$$

Thus $\psi$ is continuous at $\left(g_{1}, x_{0}\right) \in G \times X$.

Next, we shall state an open mapping theorem in this case. In this note, a group $G$ acting transitively on a metric space $X$ means that for each $x, y \in X$ there exists an element $g \in G$ with $g x=y$.

TheORem B. Let $G$ be a Polish group acting transitively on a complete metric space $X$. For each $x \in X$ the map $G \ni g \rightarrow \psi(g, x)=g \cdot x \in X$ is open. 
Proof. Let $B(x, \delta)=\{y ; d(x, y)<\delta\}$, that is, the open ball with center $x$ and radius $\delta>0$. Let $x_{0} \in X$ be fixed. We shall prove that for any neighbourhood (nbd in short) $U$ of the unit $e$ of $G$, there exists an open ball with

$$
\overline{U x}_{0} \supset B\left(x_{0}, \delta\right)
$$

for some $\delta>0$.

If this is proved, then we can prove the theorem as follows: for any $x_{0} \in X$ and for any nbd $U_{0}$ of the unit $e$ of $G$, there exist $\varepsilon_{0}>0$ and $B\left(x_{0}, \varepsilon_{0}\right) \subset$ $\overline{U_{0} x_{0}}$. Now, by induction we will construct two types of decreasing sequences of nbds of $e: U_{n}$ and $V_{n}$ for a given $U_{0}$. We want to prove that any point $y_{0}$ of $B\left(x_{0}, \varepsilon_{0}\right)$ is contained in $\bar{U}_{0}^{4} x_{0}$.

There exists $V_{0} \subset U_{0}$ such that $V_{0}=V_{0}^{-1}$ (symmetric), $V_{0}^{2} \subset U_{0}$ and $\overline{V_{0} y_{0}} \subset B\left(x_{0}, \varepsilon_{0}\right)$. Choose $\delta_{0}>0$ such that

$$
\overline{V_{0} y_{0}} \supset B\left(y_{0}, \delta_{0}\right) \text { and } \delta_{0}<(1 / 2) \varepsilon_{0} \text {. }
$$

Then there exists $g_{0} \in U_{0}$ with $g_{0} \cdot x_{0}=x_{1} \in B\left(y_{0}, \delta_{0}\right)$. Choose a nbd $U_{1}$ of $e$ such that

$$
\overline{U_{1} x_{1}} \subset B\left(y_{0}, \delta_{0}\right) \text { with } U_{1}=U_{1}^{-1}, U_{1}^{2} \subset U_{0}
$$

and

$$
\left.d\left(U_{1} g_{0}\right) \text { (= diameter of } U_{1} g_{0}\right) \leq(1 / 2) \varepsilon_{0} .
$$

Choose further $0<\varepsilon_{1}<(1 / 2) \varepsilon_{0}$ so that

$$
\overline{U_{1} x_{1}} \supset B\left(x_{1}, \varepsilon_{1}\right) \text {. }
$$

Then, there exists $h_{0} \in V_{0}$ such that

$$
y_{1}=h_{0} y_{0} \in B\left(x_{1}, \varepsilon_{1}\right) \text {. }
$$

Next, choose $V_{1}$ such that $V_{1}=V_{1}^{-1}, V_{1}^{2} \subset V_{0}$, with

$$
\overline{V_{1} y_{1}} \subset B\left(x_{1}, \varepsilon_{1}\right) \text { and } d\left(V_{1} h_{0}\right)<(1 / 2) \delta_{0} \text {. }
$$

Then there exists $0<\delta_{1}<(1 / 2) \delta_{0}$ such that

$$
\overline{V_{1} y_{1}} \supset \boldsymbol{B}\left(y_{1}, \delta_{1}\right) \text {. }
$$

Continue this process to get $\left\{U_{n}\right\},\left\{V_{n}\right\},\left\{\varepsilon_{n}\right\},\left\{\delta_{n}\right\},\left\{g_{n}\right\},\left\{h_{n}\right\},\left\{x_{n}\right\}$, and $\left\{y_{n}\right\}$ such that

$$
\begin{aligned}
& x_{n+1}=g_{n} \cdot x_{n}=\cdots=g_{n} \cdot g_{n-1} \cdots g_{0} \cdot x_{0}, \\
& y_{n+1}=h_{n} \cdot y_{n}=\cdots=h_{n} \cdot h_{n-1} \cdots h_{0} \cdot y_{0}, \\
& d\left(U_{n} g_{n-1} \cdots g_{0}\right)<(1 / 2) \varepsilon_{n-1}, \quad g_{n} \in U_{n}, \\
& d\left(V_{n} h_{n-1} \cdots h_{0}\right)<(1 / 2) \delta_{n-1}, \quad h_{n} \in V_{n},
\end{aligned}
$$


with $0<\varepsilon_{n}<(1 / 2) \varepsilon_{n-1}$ and $0<\delta_{n}<(1 / 2) \delta_{n-1}$ and

$$
\overline{U_{n} x_{n}} \supset B\left(x_{n}, \varepsilon_{n}\right) \supset \overline{V_{n} y_{n}}, \quad x_{n+1}=g_{n} \cdot x_{n} \in B\left(y_{n}, \delta_{n}\right) \text {. }
$$

Let $\lim _{n \rightarrow \infty} g_{n} \cdot g_{n-1} \cdots g_{n}=g$ and $\lim _{n \rightarrow \infty} h_{n} h_{n-1} \cdots h_{0}=h$. Then $g \in$ $\overline{U_{0}^{2}}, h \in \overline{U_{0}^{2}}$ and

$$
d\left(x_{n}, y_{n}\right)<\varepsilon_{n} \text { implies } g \cdot x_{0}=h \cdot y_{0} .
$$

Therefore, we have $y_{0}=h^{-1} g \cdot x_{0} \in \overline{U^{4}} x_{0}$.

From this result, we have that for arbitrary $x_{0} \in X$ and for arbitrary nbd $U$, there exists $\varepsilon>0$ with $U x_{0} \supset B\left(x_{0}, \varepsilon\right)$. Hence the proof is complete, if the following lemma is proved.

Lemma. For any nbd $U$ of $e$ in $G, \overline{U x_{0}}$ contains an open ball $B\left(x_{0}, \delta\right)$ form some $\delta>0$.

Proof. Let $\left\{g_{n}\right\}$ be a sequence of elements in $G$ such that $\bigcup_{n} g_{n} U=G$, since $G$ is a Polish group. Hence $\bigcup_{n} g_{n} U x_{0}=X$ so that there exists an $n_{0}$ such that $g_{n_{0}} \overline{U x_{0}}$ contains an open ball; hence $\overline{U x_{0}}$ contains an open ball $B(y, \delta)$ because $\overline{U x_{0}}=g_{n_{0}}^{-1} g_{n_{0}} \overline{U x_{0}}$. Choose a nbd $V$ of $e$ with $V=V^{-1}$ and $V^{2} \subset U$. Then $\overline{V x_{0}} \supset B(y, \delta)$ means that there exists $g \in V$ such that $g \cdot x_{0} \in B(y, \delta)$; hence there exists $\varepsilon>0$ such that $B\left(g \cdot \underline{\left.x_{0}, \varepsilon\right) \subset B(y, \delta) \subset ~}\right.$ $\overline{V x_{0}}$. Thus $g^{-1} B\left(g \cdot x_{0}, \varepsilon\right) \subset g^{-1} B(y, \delta) \subset g^{-1} \overline{V x_{0}} \subset \overline{V^{2} x_{0}} \subset \overline{U x_{0}}$. But $g^{-1} B\left(g \cdot x_{0}, \varepsilon\right)$ is an open set containing $x_{0}$, so that there exists $\delta_{1}>0$ such that $B\left(x_{0}, \delta_{1}\right) \subset g^{-1} B\left(g \cdot x_{0}, \varepsilon\right) \subset \overline{U x_{0}}$.

We express our thanks to the referee who improved our proof of Theorem $A$ and pointed out some errors in the first draft of this paper.

\section{References}

[1] S. Banach, Théorie des opérations linéares (Monografje Matematyczne, Vasovie-Lwow, 1932).

[2] M. Takesaki, Theory of operator algebra (in preparation).

Department of Mathematics

Hokkaido University

Sapporo, 060

Japan
Department of Mathematics

University of California

405 Hilgard Avenue

Los Angeles, California 90023 